
\title{
28 Research Suare \\ Relationship between Mindfulness and Burnout among Chinese Pilots: Multiple Mediation through Fatigue and Proactive Coping
}

\section{Yaning Guo}

Air Force Medical University

Ming Ji

Shaanxi Normal University

Zhiwei Yang

Air Force Medical University

Hui Wang (D971866783@qq.com )

Air Force Medical University

Xuqun You ( $\nabla$ youxuqun@yeah.net)

Shaanxi Normal University

\section{Research Article}

Keywords: burnout, fatigue, proactive coping, mindfulness, personal resource

Posted Date: March 8th, 2021

DOI: https://doi.org/10.21203/rs.3.rs-215544/v1

License: (c) (1) This work is licensed under a Creative Commons Attribution 4.0 International License. Read Full License

Version of Record: A version of this preprint was published at Social Behavior and Personality: an international journal on March 1st, 2022. See the published version at

https://doi.org/10.2224/sbp.11146. 


\section{Abstract}

Objective: The present study aimed to examine a possible relationship between mindfulness and burnout among Chinese civil pilots, and to investigate the mediating effects of fatigue and proactive coping.

Background: Based on the perspective of the extended job demand-resource model, the current study has extended prior research for identifying the mechanism underlying the roles of personal resource and personal stress in the association between mindfulness and burnout.

Method: A representative sample of 319 civil pilots drawn from the China Southern Airlines accomplished self-administrated questionnaires. Structural Equation Modeling (SEM) was used to test the hypothesized multiple mediation model with Mplus 8.3.

Results: Results addressed consistent evidence that mindfulness would play an important part in relieving and preventing burnout. A multiple mediation model showed that mindfulness exerted its indirect effect on burnout through fatigue and proactive coping, $\chi^{2}=940.495, d f=660, \chi^{2} / d f=1.425$, TLI $=.940, \mathrm{CFI}=.944, \mathrm{SRMR}=.045, \mathrm{RMSEA}=.037$. Moreover, fatigue mediated the effect of mindfulness on burnout $(\beta=-.074,95 \% \mathrm{Cl}=-.106$ to -.036$)$, whereas proactive coping mediated the association between mindfulness and burnout $(\beta=-.842,95 \% \mathrm{Cl}=-1.215$ to -.635$)$. Consequently, findings corroborated a predictive role of fatigue and proactive coping, both independently and jointly, in the indirect effect that mindfulness exerted effects on burnout.

Conclusion: Theoretical implications in the extended job demand-resource model and practical recommendations for mindfulness training practice and fatigue reduction program were discussed.

\section{Introduction}

With the improvement of aircraft technology and automation systems, there has been a series of human errors that have caused approximately $80 \%$ of accidents and incidents (Harris et al., 2001), such as Asiana Flight 214, the German Typhoon and Learjet collision, and Air France Flight 447. Human factors, such as mental health, may play an important part in aviation safety (Kelly \& Efthymiou, 2019). Pilots who suffered from mental distress will result in low work performance, temporary or permanent grounding of aircraft, and serious accidents or safe-related incidents (Fanjoy et al., 2010). Among mental health factors, burnout is described as a syndrome of emotional exhaustion, depersonalization, and sense of lack of personal accomplishment (Maslach et al., 1996). Research has shown that $32.6 \%$ of regional airline pilots were identified as high burnout candidates (Fanjoy et al., 2010 ), and $14 \%-33 \%$ of the Air Force drone operators reported to be suffering from high levels of exhaustion (Chappelle et al., 2014), suggesting a high prevalence of burnout among pilots. It is well established that burnout can be a major contributing factor in ineffective job performance, job dissatisfaction, and turnover intention (Ceyhun \& Ozbag, 2014; Fanjoy et al., 2010; Mengenci, 2014). Hence, it can be reasonably argued that burnout may have an adverse influence on occupational health and flight safety in aviation. 
Studies on pilot burnout have not yet focused on mindfulness that is broadly conceptualized as the process of paying attention to cultivate awareness of the experience of the moment in a nonjudgmental or non-evaluate accepting way (Brown \& Ryan, 2003). It is conceptualized as both personality trait and skill that can be cultivated through training and meditation. Research reporting decreased burnout after mindfulness training is in accordance with the strong evidence reported in meta-analysis of interdisciplinary literature that mindfulness practice is effective for reducing burnout (dos Santos et al., 2016; Luken \& Sammons, 2016). More importantly, mindfulness, measured by MAAS (Mindful Attention Awareness Scale) and FFMQ (Five Facet Mindfulness Questionnaire), was found to moderate the negative effects of work-related stress on depression, anxiety, and burnout (Taylor \& Millear, 2016; Westphal et al., 2015). Furthermore, mindfulness may serve as a resilience-promoting factor to enhance positive mental health factors such as hope, optimism, wellness, work well-being, life satisfaction, and self-esteem, but also mitigate symptoms of depression, anxiety, and burnout (Brown \& Ryan, 2003; dos Santos et al., 2016; Schultz et al., 2015; Sears \& Kraus, 2009; Westphal et al., 2015). The robust predictive effect of mindfulness training on performance-related anxiety has strengthened in high-performance combat aviation population (Meland, Fonne, et al., 2015). Although research has rarely examined the impact of mindfulness on burnout among pilots, based on findings mentioned above, it is reasonable to infer that mindfulness would be a predictive factor that can protect against and reduce risk of pilots' burnout.

A range of individual, situational and environmental risk factors have been identified as possible contributing factors to burnout among pilots. Studies have revealed that individual factors, such as flight time and crewmember position, situational factors such as work overload and adverse weather, and organizational factors such as aircraft maintenance issues and fair wages are influential factors on burnout (Fanjoy et al., 2010; Kearney, 2008). Situational factors and organizational factors explain how job demands and resources influence levels of burnout (Searle \& Lee, 2015). From this, it is reasonable to believe that pilots who have adapted poorly to rapid job demands from both flight safety and economic effectiveness may have far more burnout. From the perspective of the extended job demand-resource model, personal resources and personal stress, may account for the reasons why some are less prone to experience burnout compared others when exposed to difficult job demands and lack of job resources (Pennie et al., 2018). However, the majority of existing research with respect to pilot burnout has emphasized work-related demands such as work overload, as well as job resources such as organizational politics (Kearney, 2008), whereas research linking personal stress, personal resources and pilot burnout is in its infancy.

Research has emphasized the relationship between stress coping strategies and burnout, suggesting that level of burnout was predicted by different coping strategies (Charoensukmongkol, 2013). Proactive coping, which is defined as oriented coping strategy towards future expectations and challenges (Greenglass et al., 1999), can be regarded as a personal resource to alleviate risk of burnout (Chang \& Chan, 2015). Fatigue can serve as subjective personal stress that may be caused by performing multiple cognitive tasks for extended periods under high workload, accompanied with prolonged work hours and 
natural body-rhythm disturbances (Goffeng et al., 2019). It is noted that fatigue is a main feature and precursor of burnout syndrome, while burnout can be seen as a work-related fatigue condition

(Schaufeli \& Taris, 2005; Van et al., 2015). Fatigue can account for nearly $21 \%$ of human error in aviation accidents and incidents in both military and civil aviation (Harris et al., 2001), such as 1998 Little Rock accident. As mentioned above, a wide range of protective and risk factors for burnout have been identified as personal stress and personal resources. In order to evaluate and discriminate personal resources which are invulnerable to burnout and personal stress which is prone to burnout, the present study aimed to examine the robust impact of mindfulness on burnout, and the mediating effects of fatigue and proactive coping. Figure 1 depicted the hypothesized multiple mediation model.

\section{Mindfulness, proactive coping and burnout}

It is now well established that mindfulness training has widely used and consistently validated to the reduction of stress/strain in a comprehensive qualitative review (Eby, 2019). Meta-Analysis has evaluate trait mindfulness not only clearly correlated with burnout but also serves as a mitigation tool to treat job burnout (Luken \& Sammons, 2016; Mesmer-Magnus et al., 2017; Pennie et al., 2018). It is noted that proactive coping can eliminate and prevent stress before it occurs (Chang \& Chan, 2015; Searle \& Lee, 2015). Significantly positive associations are found between mindfulness and proactive coping in the prediction of life satisfaction (Hinterman et al., 2012). Moreover, regular mindfulness meditation practice contributes indirectly to reduced burnout and higher job satisfaction, in which different coping strategies can differently affect levels of burnout (Charoensukmongkol, 2013). Mindfulness has been considered as a prime characteristic for preventing burnout. As a consequence, it is reasonable to argue that proactive coping has an impact on enhancing one's coping skills to deal with stress, which is consistent with the protective role of mindfulness in stress management.

According to the extended job demand-resource model, coping strategy, regarded as a personal resource, is found to moderate the impact of job demands on burnout. Proactive coping is found to be positively associated with positive outcomes such as engagement, well-being, and negatively associated with burnout (Bermejo-Toro et al., 2015; Searle \& Lee, 2015). Study targeted the function of personal resources involved in burnout has indicated that the association between demands and burnout is modulated by proactive coping (Bermejo-Toro et al., 2015). It is suggested that proactive coping produces an effect on burnout and reactions to stress.

Previous research has demonstrated the effect of proactive coping in relation to mindfulness on burnout and health. It is noted that similar protective role of proactive coping is found to moderate relationship between stressors and burnout, as well as relationship between stressors and engagement (Searle \& Lee, 2015). In addition, findings about proactive coping affecting levels of burnout are consistent with the statement that proactive coping ought to be a particularly effective strategy for managing job demands. A stress management intervention provides considerable evidence that proactive coping has a mediating effect in the association between optimism and burnout to promote mental health among nurses (Chang \& Chan, 2015). Furthermore, proactive coping mediates the association between emotion appraisal and 
burnout, in which employees those with higher emotional intelligence can facilitate more proactive coping and then seem to experience symptoms of burnout less frequently than others (Nizielski et al., 2013). As mentioned above, proactive coping has an important role in the association between mindfulness and burnout.

\section{Mindfulness, fatigue and burnout}

Fatigue is a consequence of job demands, which can be conceived as a personal stress to result in symptoms of burnout (Aleksandra Basinska et al., 2014). A brief mindfulness-based intervention, which has been validated to cultivate mindfulness and reduce psychological distress, is proved to be effective for increasing mindfulness, and decreasing anxiety, depression, fatigue in workplace (Westphal et al., 2015). Besides, mindfulness practice is more frequently used for clinical application, in which mindfulness-based stress reduction is associated with significant improvements in self-reported symptoms of fatigue (Gregoire \& Lachance, 2015). Consequently, mindfulness is a significant protector for effective prevention from subjective fatigue in reducing stress.

Relationship between burnout and fatigue may be closely intertwined, suggesting an interactional association. However, job-related emotions mediate the relationship between fatigue and burnout among police officers, in which those individuals low in fatigue may be associated with subsequently reduced burnout (Aleksandra Basinska et al., 2014). Fatigue is some of exhaustion that may recover from adequate rest and sleep, suggesting that fatigue may bring about subsequent burnout. It has demonstrated that a range of benefits from mindfulness-based interventions were found in psychological and physiological outcomes including depression, anxiety, and mental fatigue. In consideration of fatigue can be identified in physical realm or mental realm, it is reasonable to suppose that burnout might be a consequence of pilots' fatigue due to longer exposure to flying.

Burnout and fatigue are highly prevalent in a demanding and high-stress environment, especially for safety-related occupations like civil pilots. Measures of burnout can reflect general and mental fatigue; mental fatigue would be primarily related to exhaustion. Research has examined disturbed sleep and fatigue in occupational burnout, high burnout scores are more likely to be associated with self-reported fatigue, and fatigue is regarded to interfere to the onset of emotional exhaustion and depersonalization (Raftopoulos et al., 2012). It is remarkable that low-arousal negative emotions mediate relationship between fatigue and exhaustion (Aleksandra Basinska et al., 2014). Furthermore, reduced fatigue and burnout are found after mindfulness practice, indicating that mindfulness has a similar impact on fatigue and burnout (Gregoire \& Lachance, 2015). It can be argued that mindfulness predicts risk for burnout through fatigue.

Proactive coping and fatigue can be factored into the extended job demand-resource model to make it clear that mindfulness exerts its indirect effect on burnout through fatigue and proactive coping. Research has indicated that mindfulness measures can be utilized for identifying individuals at risk of psychological distress, such as anxiety, depression and burnout (Luken \& Sammons, 2016; Westphal et al., 2015). Taking into account the potential risk of pilots' burnout for flight safety, it is significant 
important to ascertain the crucial role of mindfulness on burnout, along with recommendations for prevention and intervention, and to confirm the possible mediating role of fatigue and proactive coping in the preventative effect of mindfulness on burnout.

\section{Methods}

\section{Participants}

Three hundred and nineteen civil pilots were recruited from the China Southern Airlines, ranging from 24 to 49 years of age $(M=31.31, S D=6.24)$, to provide information on individual demographics, mindfulness, fatigue, proactive coping, and burnout. The flight experience of respondents ranged from 860 hours to 48,000 hours $(M=14,794, S D=9,573)$. In order to dispel pilots' misgivings and to enhance the validity of the measurement, respondents were asked to answer the items voluntarily and anonymously, as well as to be informed that their responses would have no effect on evaluation. All methods were carried out in accordance with relevant guidelines and regulations. Informed consent was obtained from all study subjects, and the study design was approved by the appropriate ethics review board of Shaanxi Normal University.

\section{Measures}

Mindful Attention Awareness Scale (MAAS)

A Chinese translated version of MAAS (Deng et al., 2012), which was originally developed by Brown and Ryan (2003), is a 15-item self-report questionnaire that assesses the degree to which one is attentive to the present moment in one's daily experience. It is rated on a 6-point Likert scale ranging from 1 (almost always) to 6 (almost never). A sample item includes, "I find it difficult to stay focused on what's happening in the present". It has already been validated in the Chinese language to show acceptable reliability (Deng et al., 2012). The coefficient of internal consistency in the current sample was good ( $a=$ 0.968).

Proactive Coping Scale (PCS)

The proactive coping scale (subscale of Proactive Coping Inventory) is designed to measure proactive coping (Greenglass et al., 1999). It has been validated and widely used in Chinese context (Gan et al., 2007). An example is "After attaining a goal, I look for another, more challenging one". It comprises 8 questions presented in a 6-point Likert forma, ranging from 1 (not at all true) to 6 (completely true). In this study, the internal consistency was low, with a Cronbach's alpha of 0.947 .

The mental Fatigue Scale

The mental fatigue subscale from Fatigue Scale was used to measure the degree of fatigue, which comprises 6 items ranging from 0 (no) to 1 (yes). Questions pertaining to self-reported fatigue evaluate the existence and severity of mental fatigue. Sample item is "Do you have difficulty concentrating?" The 
highest score of fatigue is 6 , with a higher score indicating more serious mental fatigue (Chalder et al., 1993). The internal consistency in this study was acceptable, $a=0.642$.

Maslach Burnout Inventory - General Survey (MBI-GS)

The validated version of the Maslach Burnout Inventory (Maslach et al., 1996) comprises of three subscales which are as follows: emotional exhaustion (EE, 5 items), depersonalization (DP, 4 items) and low personal accomplishment (PA, 6 items), with a high validity and reliability in Chinese context (Wu et al., 2012). Burnout in this study was measured with EE and DP. It includes questions such as "I feel fatigued when I have to get up in the morning to face another day on the job". The items are rated on a 7point Likert-type scale, ranging from 0 (never) to 6 (every day) according to how often the statement is experienced. Hence, high scores in EE and DP scale denote higher degrees of burnout. In the present study, the Cronbach's alpha was 0.964 for entire scale, 0.943 for EE, 0.933 for DP, respectively.

\section{Data Analysis}

Descriptive analyses, Pearson correlations among study variables were performed with SPSS 22.0. Structural equation modeling (SEM) was used to test hypothesis model with Mplus 8.3, in which the maximum likelihood estimation method was employed. Measurement model and common method test were validated prior to examining path analyses. In structural equation model, model fit was evaluated using indices such as GFI, NFI, TLI, CFI, RMR, RMSEA, in which GFI, NFI, TLI and CFI greater than .90 and SRMR and RMSEA less than 08 indicated a good model fit. " $\chi 2 / d f$ " was used to assess model fit for the reason that statistic was sensitive to sample size, with ratio of less than 3 indicating an acceptable model fit.

\section{Results}

\section{Descriptive statistics}

Descriptive statistics, comparison between groups among observed variables were displayed in Table 1 . Items of burnout were coded both as continuous variable for path analysis and categorical variable for descriptive statistics. According to cutoffs suggested by Maslach et al. (1996), which was classified with high, intermediate and low levels of burnout, burnout cases were defined as having a score higher than $75_{\text {th }}$ percentile on EE and DP. The cutoff point was 3 for EE, 2.75 for DP in the present study, with $25.39 \%$ (81) pilots high in EE, $27.90 \%$ (89) pilots high in DP. Hence, a total of 98 airline pilots (30.72\%) met the defined burnout criteria. In addition, about 32.29\% (103) pilots reported one half (3 symptoms) and above symptoms of fatigue.

There were significant differences among relationship status in burnout, whereas no specific demographics (e.g. position and relationship status) predicted fatigue. More specifically, married pilots $(M=1.20, S D=1.29)$ reported higher levels of burnout than single pilots $(M=1.61, S D=1.32), t=-2.65, p<.01$. Undoubtedly, age was positively associated with flight experience, $r=.321, p<.001$. 
Mean scores, standard deviations, and Pearson correlations were shown in Table 2. As can be seen, all focal variables were significantly correlated. Mindfulness was negatively associated with fatigue, $r=-.371$, $p<.001$, and burnout, $r=-.832, p<.001$, while positively related to proactive coping, $r=.839, p<.001$. As hypothesized, burnout was positively correlated with fatigue, $r=.477, p<.001$, whereas inversely related to proactive coping, $r=-815, p<.001$. Fatigue was negatively associated with proactive coping, $r=-.378$, $p<.001$. In brief, high levels of mindfulness and proactive coping may be associated with low levels of fatigue and burnout among civil pilots.

\section{Measurement model}

Confirmatory factor analysis (CFA) test was performed to assess the fit of our theorized four-factor model. Reliabilities, Validity and correlations were provided in Table 3. Bootstrapped confidence intervals that do not contain zero signify that an effect is statistically significant. Subsequent interpretation will be made using this criterion. Specifically, the reliabilities reported on the diagonal show strong internal consistency across all measures. The patterns of correlations are consistent with both theory and previous research. The correlation results of variables are as expected. Results indicated that each latent construct produced a good fit to the data. Factor loadings for each latent construct also indicated that the items loaded well onto their respective constructs.

The measurement model comprised of four latent constructs. All loadings of item on their constructs were statistically significant at $p<0.001$ and over 0.426 . Especially, $95 \%$ bias-corrected bootstrap Cls for the relative indirect effects that do not straddle zero, indicating that mindfulness indirectly influence burnout through fatigue and proactive coping. This supports a claim that fatigue and proactive coping as mediators of the effect of mindfulness on burnout. The overall fit of the measurement model was good, and these indices were acceptable, $\chi^{2}=941.329, d f=659, \chi^{2} / d f=1.428, \mathrm{TLI}=.939, \mathrm{CFI}=.943, \mathrm{SRMR}=$ .045 , RMSEA $=.037$. Given the acceptable results in the measurement model, the path model was tested to examine the associations among the latent variables.

Common Method Variance should be tested because all data were based on self-report. A single factor approach of Harman was carried out to check the results for common method bias, from which we can assess quality of the measurement model. We added one method factor that loaded on all indicators on the basis of measurement model, to examine the hypothesis testing "single factor explaining all variations". The model did not converge. Then we tested an alternative model which allowed all indicators to load on one single factor rather than loaded on the four latent factors. Results showed that compared to original model, this model had significantly worse model fit $\chi^{2}=1996.582, d f=665, \chi^{2} / d f=$ $3.002, \mathrm{TLI}=.717, \mathrm{CFI}=.732, \mathrm{SRMR}=.068, \mathrm{RMSEA}=.079$. As a consequence, it was suggested that the observed relationships among focal variables should not be solely attributed to common method variance.

\section{Structural Equation Model}


In order to examine multiple mediation effects simultaneously, the direct, indirect, and total effects including bias-corrected bootstrapped confidence intervals for mediation effects were assessed in Structural equation modeling (SEM). Tables 4 display parameter estimates and pairwise contrasts for specific indirect effects. The structural model provides a good fit to the data. Significant specific indirect effects were found in models transmitting the effects of mindfulness on burnout, with further modelling suggesting these effects could be qualified with reference to fatigue and proactive coping. The effects of mindfulness on burnout through fatigue and proactive coping were examined. The path model has suggested that mindfulness exerts an effect on burnout via fatigue and proactive coping, $\chi^{2}=940.495, d f$ $=660, \chi^{2} / d f=1.425, \mathrm{TLI}=.940, \mathrm{CFI}=.944, \mathrm{SRMR}=.045, \mathrm{RMSEA}=.037$.

To test whether fatigue and proactive coping mediated associations between mindfulness and burnout. The association between mindfulness and burnout were significantly related to two mediators: fatigue ( $\beta$ $=-.074,95 \% \mathrm{Cl}=-.106$ to -.036$)$ and proactive coping $(\beta=-.842,95 \% \mathrm{Cl}=-1.215$ to -.635$)$. The indirect effects through the other two mediators are significant. The direct effect of mindfulness on burnout was not significant $(\beta=-.096,95 \% \mathrm{Cl}=-.346$ to .263$)$. The indirect effects of mindfulness on burnout through fatigue and proactive coping were evaluated in proposed structural relationships among study variables. On the basis of the results, it could be concluded that fatigue and proactive coping fully mediated the association between mindfulness and burnout.

Bootstrapping indirect effects and $95 \%$ confidence intervals for multiple mediation model were conducted to compare mediating roles of fatigue and proactive coping. Bootstrapping analyses indicated that mindfulness was determined to have an indirect effect on burnout, in which fatigue and proactive coping mediated the effect of mindfulness on burnout. Using percentile bootstrap and bias-corrected bootstrapping procedures to evaluate mediating effects, results indicated that indirect effect which was mediated by proactive coping had more power than those of fatigue (indirect effect of proactive coping minus indirect effect of fatigue $=-.768,95 \% \mathrm{Cl}=-1.211$ to -.631 ). As described in Table 4 , findings supported that the mediating role of proactive coping was significantly larger than fatigue.

\section{Discussion}

Consistent with prior research, the current study addressed a moderate level of burnout among Chinese civil pilots (Fanjoy et al., 2010). Contrary to the finding that single nurses experienced more emotionally exhausted than married nurses (Sahraian et al., 2008), our results showed that married pilots suffered from higher levels of burnout than single pilots. As with a nurse, a pilot must encounter the same scheduling problems as well as the enormous work pressure that demands substantial responsibility in a challenging environment. Perhaps the opposite conclusion is probably attributed, at least in part, to the argument that the biggest difference between nurses and pilots is not the nature of the work, but the discrepancy between one being a female-dominated profession and the other a male-dominated profession. Another plausible alternative explanation could be that married pilots may face more complicated non-work related pressure or work-family conflicts than others. It may also be partly due to 
the fact that some of the pilots may spend a fair amount of time away from home as part of their flight schedule.

Burnout, regarded as a risk factor for occupational health, is a major determinant of job dissatisfaction and turnover intention among pilots (Ceyhun \& Ozbag, 2014; Fanjoy et al., 2010). Exhaustion was a strong predictor that can help inform performance improvement and risk mitigation for safety among Air Force Drone Operators (Chappelle et al., 2014). Results showed that mindfulness played an important part in relieving and preventing burnout. Whereas research has examined how job demands and job resources relate to burnout among flight crewmembers and pilots (Kearney, 2008), there is surprisingly little study on how personal stress and personal resources are related to burnout. In order to ensure flight safety and economic effectiveness, it is far more important for researchers to ascertain and distinguish risk and protective factors in burnout prevention among pilots. For identifying personal stress and personal resources in burnout prevention, the present study examined the robust protective effect of mindfulness on burnout, and the indirect effect of mindfulness on burnout through causally linked multiple mediators of fatigue and proactive coping.

Consistent with previous findings suggesting that mindfulness acts as a buffer against burnout (Taylor \& Millear, 2016), the current study demonstrated the protective effect of mindfulness on burnout among civil pilots. Mindfulness had a positive effect on pilot burnout, corroborating prior findings that mindfulness training has been consistently validated to moderate the adverse effects of job-related stress on burnout (dos Santos et al., 2016; Luken \& Sammons, 2016). Consistent with previous studies suggesting that mindfulness made a strong contribution to protecting against the onset of burnout (Luken \& Sammons, 2016; Taylor \& Millear, 2016), the current study revealed the protective effect of trait mindfulness on burnout.

Prior research has indicated that proactive coping mediated the relationship between stress and engagement, suggesting protective roles in stress management for these purposeful coping approaches (Gan et al., 2007). The results indicated that mindfulness had an impact on proactive coping and burnout, suggesting that mindfulness had a positive effect on coping strategy (Charoensukmongkol, 2013). As predicted, proactive coping were significantly associated with burnout, in which it facilitated adaptive regulation of potential challenges in helping one to be insusceptible to burnout. Similar to previous research finding that coping strategies mediate positive effects of mindfulness on burnout (Charoensukmongkol, 2013), the present study addressed the mediating role of proactive coping in the association between mindfulness and burnout. The robust relationship of higher levels of proactive coping with lower levels of burnout is mainly due to the fact that coping strategies facilitate positive outcomes and protect against the risk of burnout symptoms (Searle \& Lee, 2015). Our work has extended previous research to ascertain the important role of mindfulness and proactive coping in protecting against burnout among pilots.

According to the nature of airline transportation, the occupational stress-related phenomenon that pilots may be more prone to suffer from sleep deprivation, fatigue and burnout. Research regarding 
Mindfulness-based intervention demonstrated the availability and good efficacy of mindfulness training for reducing symptoms of fatigue and burnout (Luken \& Sammons, 2016; Westphal et al., 2015). Furthermore, The current study confirmed that fatigue mediated the effect of mindfulness on burnout, which was consistent with previous studies suggesting that individuals low in fatigue may be associated with subsequently reduced burnout (Aleksandra Basinska et al., 2014). In view of the great significance of fatigue and burnout on flight performance, our findings contribute to the argument that mindfulness is crucial for pilots to protect against fatigue and burnout. Mindfulness made a contribution to protect against the onset of burnout and fatigue. Thus, our findings highlighted the value of mindfulness, and potential benefits of mindfulness in diminishing fatigue and subsequent burnout.

\section{Theoretical Implications in Job Demand-Resource Model}

The current study attempted to confirm the influential role of personal stress and personal resources in burnout, by conducting a correctional cross-sectional study to assess the relationships between mindfulness, fatigue, proactive coping, and burnout among pilots. According to the extended job demand-resource model (Pennie et al., 2018), personal resources act as positive self-evaluations to moderate effects of job demands, and to predict performance, job satisfaction, and burnout (Chang \& Chan, 2015; Charoensukmongkol, 2013). Therefore, personal factors can help explain why certain individuals suffer from burnout or why certain individuals have more symptoms of burnout than others (Searle \& Lee, 2015). Though enormous studies concerned about the roles of work-related characteristics and resources on burnout, our results confirmed the possible mechanism that mindfulness had an important preventative effect on burnout, in which the effect was probably triggered by personal resources to exert effect to buffer against stress. It is suggested that coping strategy, regarded as a valued resource, may help individuals take appropriate actions to anticipate and deal with potential challenges (Bermejo-Toro et al., 2015; Searle \& Lee, 2015). In hence, it seems that individuals engaged in proactive coping to offset potential problems may be less vulnerable to burnout as a result of the fact that proactive coping integrates a positive approach of self-regulatory goal attainment to buffer against demands and stress to burnout (Bermejo-Toro et al., 2015; Charoensukmongkol, 2013; Searle \& Lee, 2015). However, the robust effect of mindfulness in reducing burnout was rapidly decreased, when taking into account the mediating roles of fatigue and proactive coping. The results made a contribution to the extended job demand-resource model that proactive coping acts as a personal resource to play a protective role in preventing burnout, while fatigue was regarded as personal stress to have an adverse effect on burnout. The results were also consistent with previous research with regard to the effectiveness of mindfulness training practice and fatigue reduction program.

\section{Practical Implications for mindfulness intervention and burnout reduction}

In consideration of the fact that the approach to train an excellent civil pilot is usually difficult, expensive, and time-consuming, it is extremely important to select the most suitable candidate and then train them well to ensure flight safety. The nature of the flight profession and high demand for safety may bring about high job stress, forcing pilots to withdraw psychologically with tremendous pressure, inefficiency, 
low organizational commitment, and risk of flight accidents. The current study has extended previous research suggesting that mindfulness, which protects against experiencing the negative effects of stress, helps to increase resistance to pilot burnout. We also found a mediating role of fatigue and proactive coping in the indirect effect of mindfulness on burnout. The results were undoubtedly consistent with previous research in which mindfulness was attested to have a beneficial role not only in promoting wellbeing, but also in decreasing anxiety, depression, and burnout (Charoensukmongkol, 2013; dos Santos et al., 2016; Westphal et al., 2015). Additionally, the 4-month Mindfulness training has been validated to cultivate trait mindfulness in Military Helicopter personnel, as measured by FFMQ (Meland, Ishimatsu, et al., 2015). Our findings highlighted the value of personal resources, and the potential practical benefits of utilizing mindfulness measures in pilot training programs for identifying pilots at risk of burnout.

Mindfulness practice is frequently used for practical applications. Mindfulness-based stress reduction is associated with a significant decrease in burnout rates (Luken \& Sammons, 2016). As previously mentioned, mindfulness interventions elevate proactive coping and reduce fatigue, thus making employees less likely to experience burnout. It is not surprising then that mindfulness training combined with proactive coping can exert major protective effects on the occurrence and development of burnout. The current study bridged the research gap that could have implications for burnout prevention, making a thorough inquiry about the mechanism of how mindfulness can affect burnout. When considering stress management training in practice, the significant roles of proactive coping to modulate the effect of mindfulness on burnout among pilots should not be neglected.

The current study give important insight into how future fatigue and burnout prevention can be more targeted. It may have particular applied implications as training to improve the level of mindfulness has previously been shown to have stress-reducing effects in aviators, while the mechanisms behind the effects is less known. The current study extended previous research to suggest that mindfulness, which played a predictive role to modulate negative effect of stress, would help to increase resistance to pilots' fatigue and burnout. We demonstrated the robust role of mindfulness in burnout reduction, and also assessed the mediating role of fatigue and proactive coping in the indirect effect of mindfulness on burnout. The results were undoubtedly consistent with previous research that mindfulness was attested to have a beneficial role not only in promoting well-being but also in decreasing anxiety, depression and burnout (Westphal et al., 2015). Additionally, it is also well noted that mindfulness practice is the most widely used intervention to mitigate negative effects of stress on fatigue (Gregoire \& Lachance, 2015). As previously mentioned, mindfulness intervention can not only reduce fatigue but also elevate proactive coping, thus make employees less likely to experience burnout. It is not surprising then that mindfulness training combined with proactive coping can exert major protective effects on the occurrence and development of burnout. Considering combined roles of fatigue and proactive coping, mindfulness indirectly affected burnout through two mediators without direct effect on burnout. It may have particular applied implications as training to improve the level of mindfulness has previously been shown to have stress-reducing effects in aviators. The current study bridged research gap that could have implications for burnout prevention and Fatigue Risk Management System (FRMS), making a thorough inquiry about mechanism of how does mindfulness work in fatigue and burnout reduction.

Page $12 / 18$ 


\section{Limitations and Directions for Future Research}

The present study has several limitations that require mentioning. Firstly, correlation coefficients among the study variables were extremely high. This may be partially attributed to socially desirable responding and faking, a relatively small homogeneous sample, and inappropriate measurement instruments. Secondly, self-report measures have the potential for multicollinearity issues and observational error. Especially, fatigue/sleepiness has clearly been demonstrated to have a number of cognitive effects that may influence performance and lead to accidents. Further research with respect to pilot fatigue can focus around fatigue and sleepiness, which is typically measured with Samn-Perelli Crew Status Check (Caldwell \& Caldwell, 2004) and Karolinska Sleepiness Scale (Wu et al., 2016). Furthermore, due to the various limitations such as the research sample, sample size, and cross-sectional design, caution is needed with regard to generalizability of the findings. We cannot draw conclusions about causality from the correlation data based on a cross-sectional survey. In addition, research has demonstrated that fatigue/sleepiness have a number of cognitive effects that may influence performance and lead to accidents. Further data are needed to draw inferences concerning the relationship with aviation accidents. More objective measures or instruments should be used or developed to better assess the negative factors that are particularly associated with accident proneness, self-perceived poor performance, and unhealthy mental conditions among pilots. Additionally, longitudinal studies that can better explore and ascertain evidence of causal relationships rather than cross-sectional correlations should be conducted. More importantly, practical research should be performed to further investigate causation among positive psychological factors, mental health, job performance, and safety behaviors in the aviation domain.

\section{References}

Aleksandra Basinska, B., Wiciak, I., \& Maria Dåderman, A. (2014). Fatigue and burnout in police officers: the mediating role of emotions. Policing: An International Journal of Police Strategies \& Management, 37(3), 665-680. https://doi.org/10.1108/pijpsm-10-2013-0105

Bermejo-Toro, L., Prieto-Ursúa, M., \& Hernández, V. (2015). Towards a model of teacher well-being: personal and job resources involved in teacher burnout and engagement. Educational Psychology, 36(3), 481-501. https://doi.org/10.1080/01443410.2015.1005006

Brown, K. W., \& Ryan, R. M. (2003). The benefits of being present: mindfulness and its role in psychological well-being. Journal of Personality and Social Psychology, 84(4), 822-848. https://doi.org/10.1037/0022-3514.84.4.822

Caldwell, J., \& Caldwell, J. (2004). Fatigue in Aviation: A Guide to Staying Awake at the Stick. Aviation, Space and Environmental Medicine(No.11), 1012.

Ceyhun, G. C., \& Ozbag, G. K. (2014). Does Burnout Working Conditions Affect Marine Pilots' Job Satisfaction and Turnover Intentions? A Study of Turkish Marine Pilots. International Journal of 
Academic Research in Business and Social Sciences, 4(3), 301-312. https://doi.org/10.6007/IJARBSS/v4$\mathrm{i} 3 / 708$

Chalder, T., Berelowitz, G., Pawlikowska, T., Watts, L., Wessely, S., Wright, D., \& Wallace, E. P. (1993). Development of a fatigue scale. Journal of Psychosomatic Research, 37(2), 147-153. https://doi.org/10.1016/0022-3999(93)90081-P

Chang, Y., \& Chan, H. J. (2015). Optimism and proactive coping in relation to burnout among nurses. Journal of Nursing Management, 23(3), 401-408. https://doi.org/10.1111/jonm.12148

Chappelle, W., McDonald, K., Prince, L., Goodman, T., Ray-Sannerud, B. N., \& Thompson, W. (2014). Assessment of Occupational Burnout in United States Air Force Predator/Reaper "Drone" Operators. Military Psychology, 26(5-6), 376-385. https://doi.org/10.1037/mil0000046

Charoensukmongkol, P. (2013). The contributions of mindfulness meditation on burnout, coping strategy, and job satisfaction: Evidence from Thailand. Journal of Management \& Organization, 19(05), 544-558. https://doi.org/10.1017/jmo.2014.8

Deng, Y.-Q., Li, S., Tang, Y.-Y., Zhu, L.-H., Ryan, R., \& Brown, K. (2012). Psychometric Properties of the Chinese Translation of the Mindful Attention Awareness Scale (MAAS). Mindfulness, 3(1), 10-14. https://doi.org/10.1007/s12671-011-0074-1

dos Santos, T. M., Kozasa, E. H., Carmagnani, I. S., Tanaka, L. H., Lacerda, S. S., \& Nogueira-Martins, L. A. (2016). Positive Effects of a Stress Reduction Program Based on Mindfulness Meditation in Brazilian Nursing Professionals: Qualitative and Quantitative Evaluation. EXPLORE: the Journal of Science and Healing, 12(2), 90-99. https://doi.org/10.1016/j.explore.2015.12.005

Eby, L. T. A., Tammy D.Conley, Kate M.Williamson, Rachel L.Henderson, Tyler G.Mancini, Victor S. (2019). Mindfulness-based training interventions for employees: A qualitative review of the literature. Human Resource Management Review(No.2), 156-178.

Fanjoy, R. O., Harriman, S. L., \& Demik, R. J. (2010). Individual and environmental predictors of burnout among regional airline pilots. International Journal of Applied Aviation Studies, 10(1), 15-30.

Gan, Y. Q., Yang, M. S., Zhou, Y., \& Zhang, Y. L. (2007). The two-factor structure of future-oriented coping and its mediating role in student engagement. Pers Indiv Differ, 43(4), 851-863.

https://doi.org/10.1016/j.paid.2007.02.009

Goffeng, E. M., Wagstaff, A., Nordby, K.-C., Meland, A., Goffeng, L. O., Skare, Ø., Lilja, D., \& Lie, J.-A. S. (2019). Risk of Fatigue Among Airline Crew During 4 Consecutive Days of Flight Duty. Aerospace Medicine \& Human Performance, 90(5), 466-474.

Greenglass, E., Schwarzer, R., Jakubiec, D., Fiksenbaum, L., \& Taubert, S. (1999). The Proactive Coping Inventory (PCl): a multidimensional research instrument. 20th International Conference of the Stress and 
Anxiety Research Society,

Gregoire, S., \& Lachance, L. (2015). Evaluation of a Brief Mindfulness-Based Intervention to Reduce Psychological Distress in the Workplace. Mindfulness, 6(4), 836-847. https://doi.org/10.1007/s12671014-0328-9

Harris, W. C., Sachau, D., Harris, S. C., \& Allen, R. (2001). The relationship between working conditions and commercial pilot fatigue development. Proceedings of the Human Factors \& Ergonomics Society Annual Meeting,

Hinterman, C., Burns, L., Hopwood, D., \& Rogers, W. (2012). Mindfulness: Seeking a More Perfect Approach to Coping with Life's Challenges. Mindfulness, 3(4), 275-281. https://doi.org/10.1007/s12671012-0091-8

Kearney, L. M. (2008). Examining the relationship between Part 121 air transport pilots and burnout Northcentral University].

Kelly, D., \& Efthymiou, M. (2019). An analysis of human factors in fifty controlled flight into terrain aviation accidents from 2007 to 2017. Journal of Safety Research, 69, 155-165.

https://doi.org/10.1016/j.jsr.2019.03.009

Luken, M., \& Sammons, A. (2016). Systematic Review of Mindfulness Practice for Reducing Job Burnout. American Journal of Occupational Therapy, 70(2), 1-10. https://doi.org/10.5014/ajot.2016.016956

Maslach, C., Jackson, S. E., \& Leiter, M. P. (1996). Maslach Burnout Inventory-Manual, 3rd ed. Consulting Psychologists Press: Palo Alto, CA, USA.

Meland, A., Fonne, V., Wagstaff, A., \& Pensgaard, A. M. (2015). Mindfulness-Based Mental Training in a High-Performance Combat Aviation Population: A One-Year Intervention Study and Two-Year Follow-Up. International Journal of Aviation Psychology, 25(1), 48-61.

https://doi.org/10.1080/10508414.2015.995572

Meland, A., Ishimatsu, K., Pensgaard, A. M., Wagstaff, A., Fonne, V., Garde, A. H., \& Harris, A. (2015). Impact of Mindfulness Training on Physiological Measures of Stress and Objective Measures of Attention Control in a Military Helicopter Unit. Int J Aviat Psychol, 25(3-4), 191-208.

https://doi.org/10.1080/10508414.2015.1162639

Mengenci, C. (2014). Could burnout be a reason behind airlines accident? An Emperical Research Study in Turkish Airlines Companies. European Journal of Business \& Management, 6(30), 52-62.

Mesmer-Magnus, J., Manapragada, A., Viswesvaran, C., \& Allen, J. W. (2017). Trait mindfulness at work: A meta-analysis of the personal and professional correlates of trait mindfulness. Human Performance(No.2-3), 79-98. 
Nizielski, S., Hallum, S., Schutz, A., \& Lopes, P. N. (2013). A Note on Emotion Appraisal and Burnout: The Mediating Role of Antecedent-Focused Coping Strategies. Journal of Occupational Health Psychology, 18(3), 363-369. https://doi.org/10.1037/a0033043

Pennie, E., Eleanor, H. W., Matthew, W. H., \& Bradley, J. W. (2018). Trait Mindfulness Helps Explain the Relationships Between Job Stress, Physiological Reactivity and Self-perceived Health. J Occup Environ Med, 61(1), e12-e18. https://doi.org/10.1097/JOM.0000000000001493

Raftopoulos, V., Charalambous, A., \& Talias, M. (2012). The factors associated with the burnout syndrome and fatigue in Cypriot nurses: a census report. BMC Public Health, 12(1), 457, Article 457. https://doi.org/10.1186/1471-2458-12-457

Sahraian, A., Fazelzadeh, A., Mehdizadeh, A. R., \& Toobaee, S. H. (2008). Burnout in hospital nurses: a comparison of internal, surgery, psychiatry and burns wards. Int Nurs Rev, 55(1), 62-67. https://doi.org/10.1111/j.1466-7657.2007.00582.x

Schaufeli, W. B., \& Taris, T. W. (2005). The conceptualization and measurement of burnout: Common ground and worlds apart The views expressed inWork \& StressCommentaries are those of the author(s), and do not necessarily represent those of any other person or organization, or of the journal. Work \& Stress, 19(3), 256-262. https://doi.org/10.1080/02678370500385913

Schultz, P. P., Ryan, R. M., Niemiec, C. P., Legate, N., \& Williams, G. C. (2015). Mindfulness, Work Climate, and Psychological Need Satisfaction in Employee Well-being. Mindfulness, 6(5), 971-985. https://doi.org/10.1007/s12671-014-0338-7

Searle, B. J., \& Lee, L. (2015). Proactive Coping as a Personal Resource in the Expanded Job DemandsResources Model. International Journal of Stress Management, 22(1), 46-69.

https://doi.org/10.1037/a0038439

Sears, S., \& Kraus, S. (2009). I think therefore I om: cognitive distortions and coping style as mediators for the effects of mindfulness meditation on anxiety, positive and negative affect, and hope. Journal of Clinical Psychology, 65(6), 561-573. https://doi.org/10.1002/jclp.20543

Taylor, N. Z., \& Millear, P. M. R. (2016). The contribution of mindfulness to predicting burnout in the workplace. Personality and Individual Differences, 89, 123-128.

https://doi.org/10.1016/j.paid.2015.10.005

Van, D. A., Keijsers, G., Verbraak, M., Eling, P., \& Becker, E. (2015). Level and Appraisal of Fatigue are Not Specific in Burnout. Clinical Psychology \& Psychotherapy, 22(2), 133-141.

Westphal, M., Bingisser, M. B., Feng, T., Wall, M., Blakley, E., Bingisser, R., \& Kleim, B. (2015). Protective benefits of mindfulness in emergency room personnel. Journal of Affective Disorders, 175, 79-85. https://doi.org/10.1016/j.jad.2014.12.038 
Wu, L. J., Berg, M. J. v. d., \& Ger, P. H. (2016). Subjective Measurements of In-Flight Sleep, Circadian Variation, and Their Relationship with Fatigue. Aerospace Medicine \& Human Performance(10), 869.

Wu, S., Li, H., Zhu, W., Lin, S., Chai, W., \& Wang, X. (2012). Effect of Work Stressors, Personal Strain, and Coping Resources on Burnout in Chinese Medical Professionals: A Structural Equation Model. Industrial Health, 50(4), 279-287. https://doi.org/10.1016/j.jad.2014.12.038

\section{Tables}

Table 1 Demographic characteristic of study variables.

\begin{tabular}{llllll}
\hline Demographic Variables & $N(\%)$ & Fatigue & \multicolumn{3}{l}{ Burnout } \\
\hline Position & & & & & \\
Co-pilot & $212(66.5 \%)$ & $1.77(1.64)$ & $F=.73$ & $1.56(1.35)$ & $F=2.17$ \\
Captain & $73(22.9 \%)$ & $2.04(1.67)$ & $p=.48$ & $1.35(1.29)$ & $p=.12$ \\
Instructor & $34(10.7 \%)$ & $1.85(1.71)$ & & $1.09(1.19)$ & \\
Relationship status & & & & & \\
Single & $117(36.7 \%)$ & $1.84(1.62)$ & $t=.05$ & $1.20(1.29)$ & $t=-2.65$ \\
Married & $202(63.3 \%)$ & $1.83(1.68)$ & $p=.96$ & $1.61(1.32)$ & $p=.009^{* *}$ \\
\hline
\end{tabular}

${ }^{*} p<.05,{ }^{* *} p<.01,{ }^{* * *} p<.001$

Table 2 Descriptive statistics and Correlations between mindfulness, fatigue, proactive coping and burnout.

\begin{tabular}{lllllllll}
\hline Variables & $M$ & $S D$ & 1 & 2 & 3 & 4 & 5 & 6 \\
\hline 1. age & 31.310 & 6.243 & - & & & & & \\
2. flight experience (PFE) & 14,845 & 9,570 & $.838^{* * *}$ & - & & & & \\
3. mindfulness & 4.189 & 1.258 & .056 & .025 & - & & & \\
4. fatigue & 1.828 & 1.652 & .042 & -.018 & $-.371^{* * *}$ & - & & \\
5. proactive coping & 4.624 & 1.321 & .079 & .037 & $.839^{* * *}$ & $-.378^{* * *}$ & - \\
6. burnout & 1.455 & 1.323 & -.053 & -.027 & $-.832^{* * *}$ & $.477^{* * *}$ & $-.815^{* * *}$ & - \\
\hline
\end{tabular}

Note. ${ }^{* * *}$ Correlation is significant at the 0.001 level (2-tailed), ${ }^{*}$ Correlation is significant at the 0.05 level (2-tailed).

Table 3 The Reliability, Convergence Validity and Discriminant Validity of the Latent Variables.

\begin{tabular}{|c|c|c|c|c|c|c|c|c|}
\hline \multirow[t]{2}{*}{ DIM. } & \multirow[t]{2}{*}{ ITEMS } & \multirow{2}{*}{$\frac{\text { Item Reliability }}{\text { STD. Loading }}$} & \multirow{2}{*}{$\begin{array}{l}\text { Composite Reliability } \\
\text { CR }\end{array}$} & \multirow{2}{*}{$\begin{array}{l}\text { Convergence Validity } \\
\text { AVE }\end{array}$} & \multicolumn{4}{|c|}{ Discriminate Validity } \\
\hline & & & & & $\mathrm{X}$ & M1 & M2 & $\mathrm{Y}$ \\
\hline $\mathrm{X}$ & 15 & $0.761-0.897$ & 0.968 & 0.666 & 0.816 & & & \\
\hline M1 & 6 & $0.426-0.838$ & 0.804 & 0.420 & $-.371^{* * *}$ & 0.648 & & \\
\hline M2 & 8 & $0.788-0.879$ & 0.947 & 0.693 & $.839^{* * *}$ & $-.378^{* * *}$ & 0.832 & \\
\hline $\mathrm{Y}$ & 9 & $0.810-0.902$ & 0.963 & 0.741 & $-.832^{* * *}$ & $.477^{* * *}$ & $-.815^{* * *}$ & 0.861 \\
\hline
\end{tabular}

Note. The bold diagonal is the square root of AVE, and the lower triangle is the Pearson's correlation coefficient. 
Table 4 Comparison of Specific Indirect Effect.

\begin{tabular}{|c|c|c|c|c|c|c|c|c|}
\hline \multirow[t]{3}{*}{ Estimate } & & \multirow{2}{*}{\multicolumn{3}{|c|}{ Product of Coefficients }} & \multicolumn{4}{|c|}{ BOOTSTRAP 1000 TIMES 95\%CI } \\
\hline & & & & & \multicolumn{2}{|c|}{ bias corrected } & \multicolumn{2}{|c|}{ percentile } \\
\hline & & S.E. & Est/S.E. & P-Value & Lower & Upper & Lower & Upper \\
\hline \multicolumn{9}{|l|}{ INDIRECT EFFECT } \\
\hline Indirect effect of mental fatigue (M1) & -.074 & .020 & -3.623 & .001 & -.106 & -.056 & -.106 & -.036 \\
\hline Indirect effect of proactive coping (M2) & -.842 & .197 & -4.284 & .001 & -1.215 & -.664 & -1.215 & -.635 \\
\hline TOTALIND & -.916 & .187 & -4.886 & .001 & -1.103 & -.904 & -1.278 & -.692 \\
\hline DIRECT EFFECT & -.096 & .190 & -.509 & .611 & -.346 & .263 & -.346 & .263 \\
\hline \multicolumn{9}{|l|}{ CONTRAST } \\
\hline M2-M1 & -.768 & .197 & -4.249 & .001 & -1.211 & -.659 & -1.211 & -.631 \\
\hline
\end{tabular}

Figures

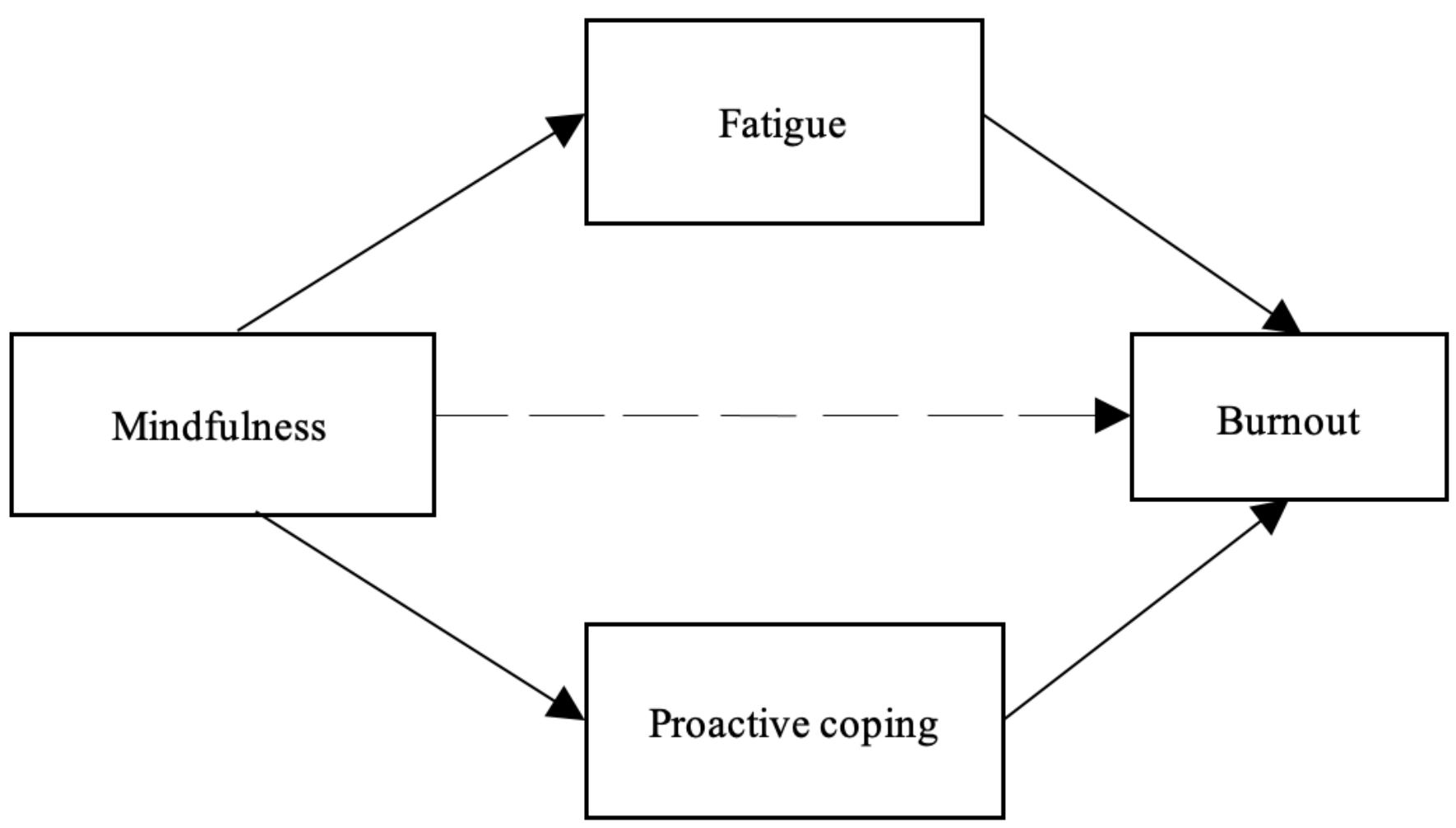

Figure 1

A multiple mediation model for the association between mindfulness and burnout. 